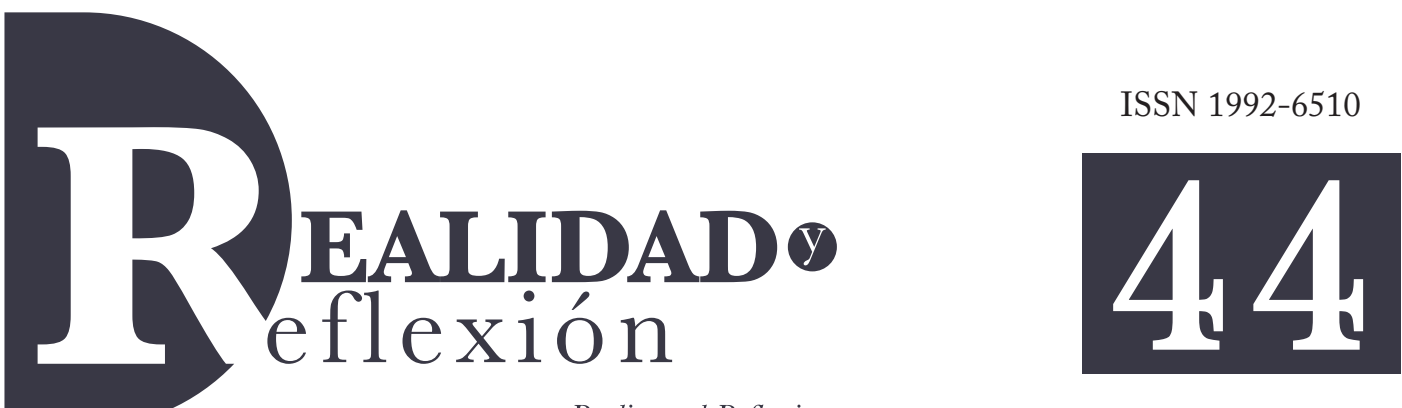

Reality and Reflection

Año 16, Nº 44, San Salvador, El Salvador, Centroamérica. Revista Semestral Julio-Diciembre 2016

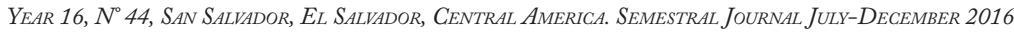

\title{
Temáticas y enfoques para la difusión del conocimiento ambiental en las universidades de El Salvador
}

\author{
Topics and approaches for the dissemination of \\ environmental knowledge in the universities of El Salvador
}

\author{
Jorge Arnoldo Valencia Granados \\ Ingeniero Industrial y Máster en Administración de Empresas \\ jorge.valencia@portallogistico.com
}

\section{RESUMEN}

E1 Salvador necesita de una difusión coherente y unificada del análisis técnico de la problemática ambiental. Esto constituye un imperativo estratégico para el país, dada su vulnerabilidad reflejada en los índices ambientales recientes.

Este artículo propone un modelo multinivel con respecto a temas ambientales, que puede ser incorporado en la educación superior local. Los niveles son el país, la industria, el sector empresarial y personal. Aunque su difusión debe cubrir todas las carreras universitarias de diferentes maneras, se propone al menos cuatro temas que deben ser explorados: Marketing Verde, Producción Más Limpia, Fuentes de Energías Renovables y Logística Inversa.

Palabras clave: medio ambiente, marketing verde, producción limpia, fuentes de energías renovables, logística inversa, EPI.

ABSTRACT

El Salvador needs a consistent dissemination and unified technical analysis of the environmental problems. This is a strategic imperative for the country given its vulnerability reflected in the recent environmental indexes.

This article proposes a multilevel model regarding to environmental issues that can be permeated in local higher education. The levels are country, industry, business and personal. Although its dissemination should cover all the university careers in different ways, it is proposed at least four topics that should be explored: Green Marketing, Cleaner Production, Renewable Energy Sources and Reverse Logistics.

Keywords: Enviroment, Green Marketing, Cleaner Production, Renewable Energy Sources, Reverse Logistics, EPI, Carbon footprint. 


\section{Introducción}

Logística inversa, marketing verde, energías renovables, producción más limpia, ecodiseño, responsabilidad social empresarial, sostenibilidad, etc. son algunos de los términos recientes que tienen como trasfondo la preocupación por detener el deterioro ambiental mundial. Cada uno de ellos exhibe una realidad compleja en la que se conjuga la utilización eficiente de los recursos naturales existentes y el fomento de la conciencia ecológicamente responsable. Indirectamente, el crecimiento económico sostenible en cada país.

Resulta paradójico que en El Salvador, con tantos problemas ambientales, la inversión en la investigación, docencia y formación en esta área sea tan escasa. Baste revisar las mallas curriculares de las instituciones de educación superior para percatarse de la falta de carreras, asignaturas o cursos libres enfocados en el tema. Como que no interesa. De acuerdo al Ministerio de Educación (MINED) y a su listado de las carreras autorizadas a ser impartidas por las instituciones de educación superior, actualizado al 11 de agosto del 2016, las universidades que cuentan con este tipo de estudio se muestran en el Cuadro n. ${ }^{\circ} 1$.

Adicional a este cuadro de datos, y utilizando la misma fuente del MINED, cabe mencionar que “...de las carreras solicitadas de la Universidad de El Salvador, para el año 2015 y de conformidad a los datos proporcionados por la institución las carreras de Maestría en Medio Ambiente y Recursos Naturales, Maestría en Gestión Integral del Agua y Licenciatura en Ecotecnología, fueron reportadas como carreras no activas, por lo que no se reportan datos para las mismas. Sin embargo, se agregaron otras afines”.

Los estudios formales ambientales, como puede constatarse en el Cuadro 1, se concentran a nivel superior de maestrías y no a nivel de pregrado, donde la población estudiantil es mucho mayor. Geográficamente, los estudios se concentran en San Salvador, la capital del país. También hay carreras en las zonas Occidental y Oriental. Paradójicamente, en el oriente, la región más deforestada solo la Universidad de E1 Salvador ofrece carreras en esta línea con 10 alumnos registrados.

¿A qué se debe todo esto? Quizá por ser un tema relativamente reciente -inicios de la década del 70- y que los conocimientos están fluyendo lentamente de la práctica a la academia, de manera independiente, y no enmarcada en lineamientos más o menos estandarizados; los temas ambientales se abordan atomizados y tímidamente a nivel nacional. La currícula es diferente, así como el abordaje. Depende mucho del docente, pero también ocurre que la toma de conciencia en los niveles escolares y de pregrado no es suficiente. La escasa matrícula en todo nivel también puede responder a una sobreestimación de la demanda a la hora de realizar el proceso de creación de la carrera en el MINED.

Pese a todo, resulta importante para el país la divulgación del conocimiento de los temas ambientales a todo nivel. Las universidades pueden contribuir significativamente a que los nuevos profesionales desarrollen criterios ambientales aplicables dentro de sus carreras. Hasta el momento, sólo están disponibles de forma no estructurada por medio de internet, 


\section{Cuadro n. ${ }^{\circ} 1$}

Estudios universitarios sobre temas ambientales en E1 Salvador

\begin{tabular}{|c|c|c|c|c|c|}
\hline Nombre de institución & Sede centro regional & n. ${ }^{\circ}$ & Carrera & n. ${ }^{\circ}$ & $\%$ \\
\hline $\begin{array}{l}\text { Universidad Católica de } \\
\text { El Salvador }\end{array}$ & Santa Ana - central & 40 & $\begin{array}{l}\text { Maestría en Gerencia y } \\
\text { Gestión Ambiental }\end{array}$ & 40 & $11.3 \%$ \\
\hline $\begin{array}{l}\text { Universidad Católica de } \\
\text { El Salvador }\end{array}$ & Santa Ana - central & 37 & $\begin{array}{l}\text { Ingeniería civil opción } \\
\text { Saneamiento Ambiental }\end{array}$ & 37 & $10.5 \%$ \\
\hline $\begin{array}{c}\text { Universidad } \\
\text { Centroamericana José } \\
\text { Simeón Cañas }\end{array}$ & La Libertad - central & 36 & $\begin{array}{c}\text { Maestría en Gestión del Medio } \\
\text { Ambiente }\end{array}$ & 36 & $10.2 \%$ \\
\hline Universidad Don Bosco & San Salvador - central & 50 & $\begin{array}{l}\text { Maestría en Gestión de } \\
\text { Energías Renovables }\end{array}$ & 50 & $14.2 \%$ \\
\hline Universidad de E1 Salvador & San Miguel - regional & 10 & Maestría en Gestión Ambiental & 10 & $2.8 \%$ \\
\hline Universidad de E1 Salvador & San Salvador - central & 55 & $\begin{array}{c}\text { Maestría en Energías Renovables y } \\
\text { Medio Ambiente }\end{array}$ & 55 & $15.6 \%$ \\
\hline Universidad de E1 Salvador & San Salvador - central & 56 & $\begin{array}{l}\text { Maestría en Gestión de } \\
\text { Recursos Hidrogeológicos }\end{array}$ & 56 & $15.9 \%$ \\
\hline \multirow[t]{2}{*}{ Universidad de E1 Salvador } & San Salvador - central & 69 & Licenciatura en Salud Ambiental & 69 & $19.5 \%$ \\
\hline & & & & 353 & $100.0 \%$ \\
\hline
\end{tabular}

Fuente: Departamento de Información Estadística del Ministerio de Educación de El Salvador

programas de televisión, congresos, seminarios, diplomados y otros. El desarrollo de las temáticas no es coherente en temas ni en la profundidad de los mismos.

A fin de dar un abordaje propositivo a esta problemática, a continuación se plantean los niveles en los cuales puede dimensionarse el enfoque de estos temas en la educación superior: nacional o nivel país, industrial, empresarial y personal. En segundo lugar, se propone cómo o en qué materias insertar los temas ambientales dentro de la currícula de Ingenierías y Licenciaturas, básicamente. Son temas técnicos, legales, económicos, financieros, etc., que requieren de una gestión. La difusión de temas ambientales debería abarcar otras carreras, pero limitaremos el enfoque a las áreas comerciales más tradicionales.
Para ello se plantean cuatro temáticas que incluso pueden constituirse en materias electivas o cursos de especialización: marketing verde, producción más limpia, fuentes de energías renovables y logística inversa. Lo importante es comenzar con algo e irlo perfeccionando. El conocimiento y el compromiso ambientales del momento, que se transmiten sólo a niveles de maestría o de manera desarticulada deben superarse. No son suficiente.

Dada la complejidad de enfoques, normativas y estándares se hace necesario unificar contenidos, criterios y selección de herramientas, para lo cual se sugiere la creación de un Observatorio Académico de Prácticas Ambientales que permita buscar sinergias entre las instituciones educativas de nivel superior. En otros países ya existen iniciativas como esta: tómese como ejemplo el 
ITBA (Instituto Tecnológico de Buenos Aires), que desarrolla anualmente, desde el 2014, los foros de sustentabilidad como plataforma para mostrar investigaciones sobre buenas prácticas. Es un esfuerzo academia - sector privado.

\section{Niveles o dimensiones del enfoque ambiental}

Lo primero que debe quedar claro es que sin medición no puede haber mejora. Hay que partir de algo y continuar su medición utilizando el mismo criterio en el tiempo. Esto constituye de entrada uno de los primeros problemas teóricoprácticos con que enfrenta la difusión del conocimiento en esta área. ¿Qué medir? ¿Cuál es el marco de referencia a utilizar? En este sentido, tómese en cuenta que el enfoque de los mismos puede ser a nivel de país, región, industria o empresa y llegar incluso hasta a nivel individual como consumidores. Todo ello permite analizar desde una perspectiva macro o micro la situación del fenómeno ambiental en nuestro país.

\section{a. Medición a nivel de país}

Comencemos con los datos estadísticos e indicadores ambientales a nivel nacional. En el año 2008, cuando las universidades de Yale y Columbia desarrollaron conjuntamente un método para cuantificar y clasificar numéricamente el desempeño ambiental de las políticas de un país. El resultado de esta colaboración es el indicador ambiental EPI (Environment Performance Index), que sirve de base comparativa del desempeño entre países.

El EPI es un indicador compuesto y complejo. Está conformado por una serie de otros indicadores de menor nivel, pero más específicos a los objetivos medibles. Tal es el caso del agua potable, calidad del agua natural, suspensión de partículas en el aire, reserva de árboles en crecimiento, emisiones industriales de $\mathrm{CO} 2$ . Todo ello, para su comprensión, se agrupa en políticas y objetivos. A nivel del ranking mundial, El Salvador ocupó la posición 97 de 180 países en el último reporte correspondiente al 2016, aunque en algunas áreas como calidad del agua, recursos hídricos, agricultura, recursos pesqueros y conservación de la biodiversidad se observen puntajes extremadamente críticos. (Ver Figura n. ${ }^{\circ}$ 1).

Pese a su aporte valioso, el EPI no es un indicador único. Organismos regionales como la Comisión Económica para América Latina y el Caribe (CEPAL), Gobiernos diversos y hasta empresas multinacionales han creado sus propias escalas tropicalizando los datos y haciéndolas congruentes con la normativa vigente a los países y a sus industrias en particular. Para citar algunos, tenemos el SINIA (Sistema Nacional de Información Ambiental), en el Perú; en México, el SNIA ; en El Salvador, el SEA; GOGREEN en DHL, y ECO Solutions en el operador logístico internacional Shenker. Tienen mucho en común, pero no son iguales.

A nivel centroamericano, como referencia regional dentro del mismo indicador EPI, Costa Rica ocupa la posición 42, seguida de Panamá (51), Guatemala (88), Honduras (89), El Salvador (97) y Nicaragua (115).

\section{b. Medición a nivel de industria}

Desde hace varios años, las empresas industriales han mostrado su interés en transformar sus 


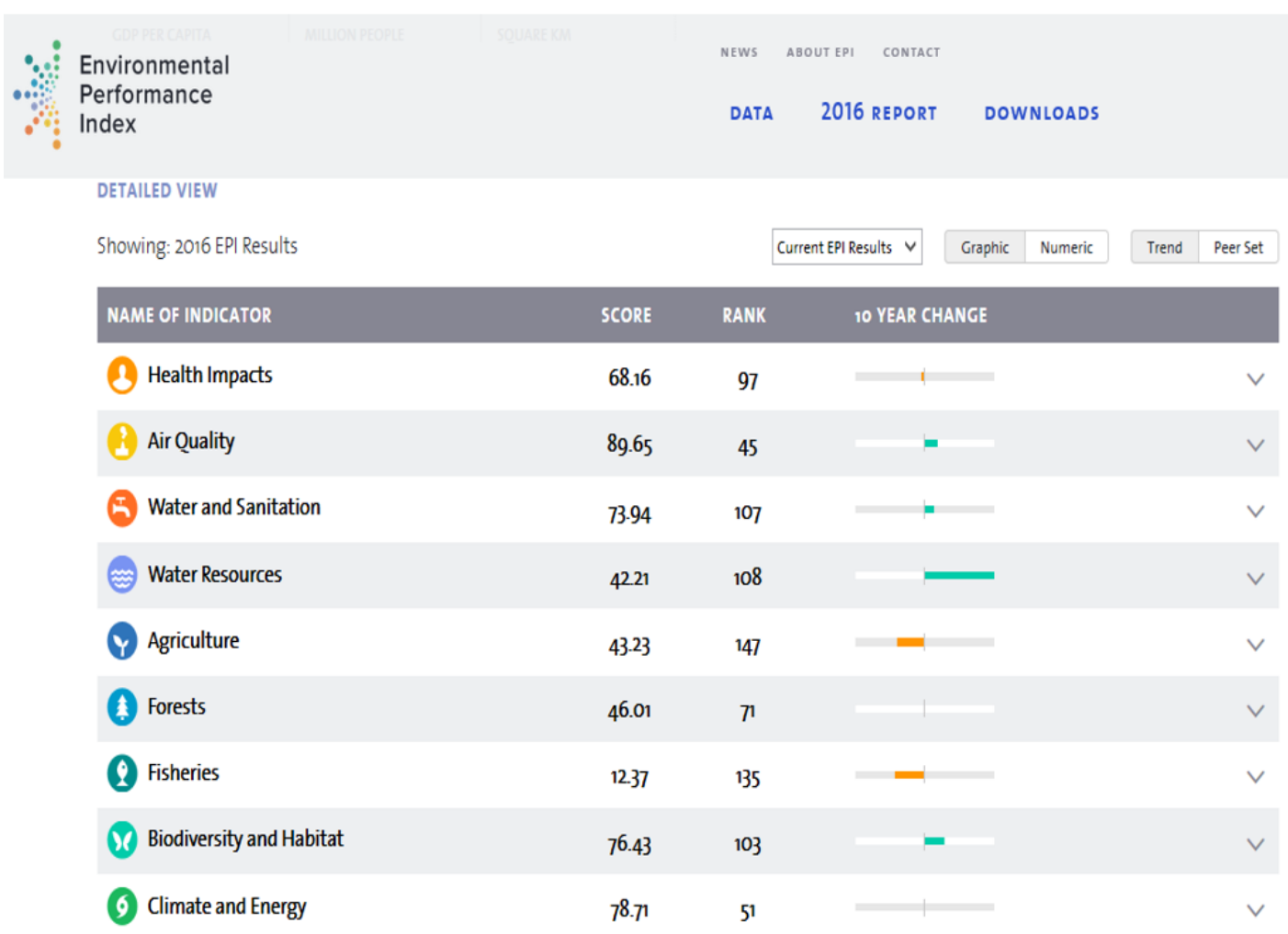

Figura n. ${ }^{\circ}$ 1. Universidad de Yale, Índice Ambiental 2016. Fuente: http//epi.yale.edu/country/el-salvador

procesos productivos para hacerlos más eficientes y amigables con el entorno. De igual manera, dicho interés se refleja también en los esfuerzos de modificar la percepción que el mercado tiene de los productos o servicios recibidos. De ahí que nace el interés de buscar la certificación de organismos internacionales que no sólo hagan lucir "verde" a las empresas, sino que también permitan medir el grado de impacto ambiental mediante una metodología científica. En muchos casos, sólo es una estrategia para vender más. No obstante, si existe una incongruencia entre lo que se publicita y lo que se hace en realidad, se cae en una práctica conocida como greenwashing o lavado verde y tarde o temprano el consumidor lo reconoce.
De nuevo enfrentamos las preguntas sobre el qué medir, cómo medir y qué metodología es la más conveniente a utilizar. En lo concerniente a la medición de la huella de carbono resultante en cada industria, concepto clave, tenemos más de una docena de ellas: Bilan Carbone, British Standard BSI PAS 2015:2011, BSI PAS 2060:2014, Ecotransit, GHG Protocol, IPCC, ISO 14067:2013, ISO 39001:2012, ISO 50001:2011, LEED 2009, SMARTRANS, etc. La huella de carbono es importante porque identifica la cantidad de emisiones de gases de efecto invernadero, que son liberadas a la atmósfera como consecuencia del desarrollo de cualquier actividad. 

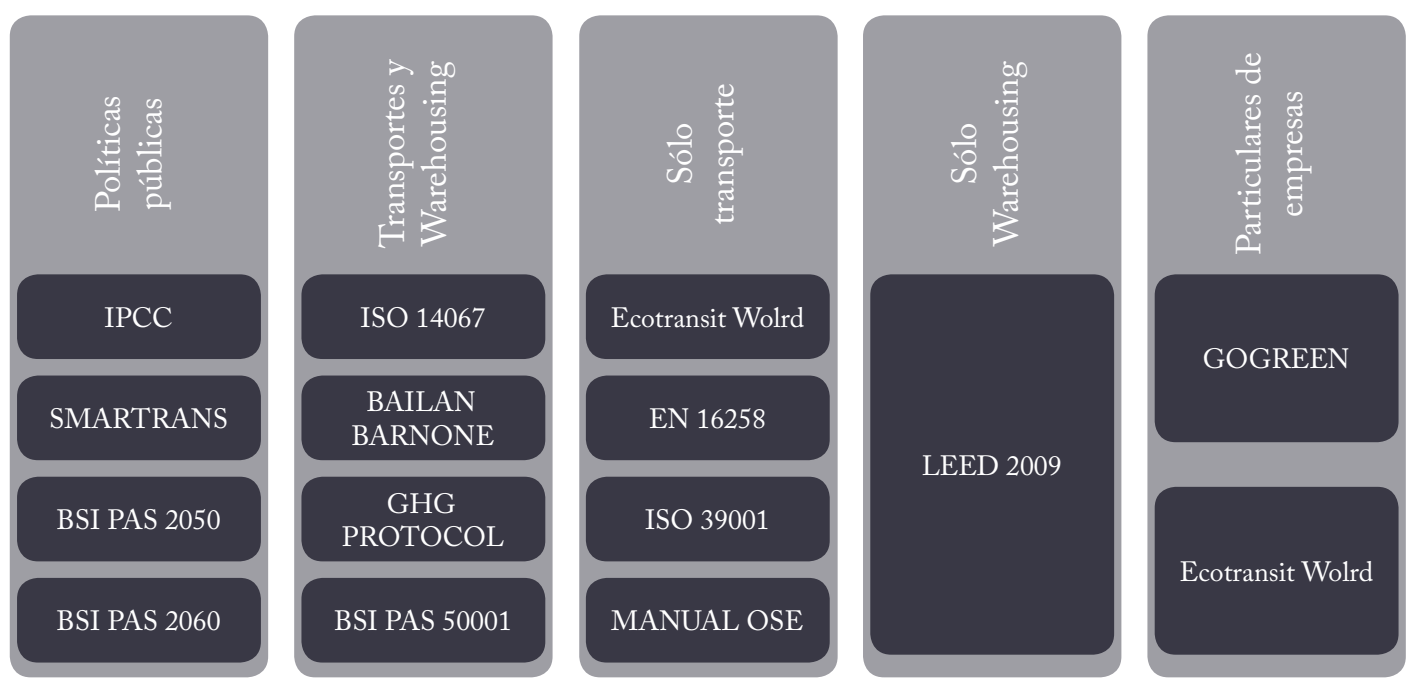

Figura . $^{\circ}$ 2. Observatorio de Logística y Sostenibilidad del ITBA, Cámara de Comercio, Industrias y Agricultura de Panamá y Secretaría Nacional de Ciencia, Tecnología e Innovación (SENACYT), Panamá. Fuente: Rodolfo Fiadone

En la Guía para el cálculo de la huella de carbón y para la elaboración de un plan de mejora de una organización, publicada en el año 2015 por la Oficina Española de Cambio Climático. Ministerio de Agricultura, Alimentación y Medio Ambiente, se detalla la metodología de cálculo de la huella de carbono a nivel empresarial, utilizando Bilan Carbone como referencia. En el mismo documento se observa que el concepto, si bien es cierto que puede tener diferentes deficiencias y complicaciones en su aplicación, nos permite identificar todas las fuentes de emisiones de GEI y establecer, fundado en este conocimiento, medidas efectivas de reducción. (Ver Figura n. ${ }^{\circ}$ 2).

Todas las normas planteadas en la Figura n. 2 comparten su enfoque en calcular lo relacionado al proceso de producción. Lo que no es sencillo y difieren entre ellas, es el alcance desde dónde comienza y hasta dónde termina dicho proceso. Algunas normas cuestionan los factores de segundo orden, como lo pueden ser la fuente de obtención de la energía utilizada, la huella de carbono de los proveedores y hasta del transporte de los empleados. Ya en la práctica, para que tenga cierta lógica, comparar la huella de carbono de un producto con otro, no nos sirve de mucho, a menos que sepamos cómo se ha calculado en cada caso. Por esta razón, organizaciones como el SMART FREIGHT CENTRE, de Europa, trabajan en unificar los procedimientos y factores de emisión utilizados en los cálculos numéricos.

Aparte de los procesos de manufactura en sí, el transporte tiene una contribución muy importante en las emisiones contaminantes de una empresa. Existen buenas prácticas y normativas para regular la emisión de gases contaminantes del transporte de carga, tales como 
conducción eficiente, mejorar la aerodinámica del vehículo de transporte, mantenimiento adecuado, planificación de rutas, control de báscula y otros.

Se observará que el tema de la industria es muy especializado y de complejidades muy asimétricas. Para citar un último caso, tenemos la normativa europea ROM 5.1 para el análisis de calidad de las aguas litorales portuarias. Dicha temática se enseña en la Universidad de Cantabria, España, en las carreras relacionadas con las ciencias del mar, desde hace muchos años. En nuestro país se desconoce y no hay regulación al respecto.

\section{c. Medición a nivel de empresa}

Las certificaciones ambientales ISO y los ECOSELLOS son también instrumentos que permiten a la empresa validar sus procesos internos, que buscan minimizar los impactos ambientales que suponen cualquier transformación productiva.

En El Salvador, la norma más utilizada en este sentido ha sido la de la Organización Internacional para la Estandarización ISO14001, que se comenzó a publicar desde el año 1996. Con dicha certificación medioambiental se da a entender que la empresa es objeto de las evaluaciones y los controles que se establecen conjuntamente con la empresa certificadora. Algunas de las empresas certificadas bajo esa norma son CEL, Bayer, AMANCO, Kimberly Clark, etc.

Aparte de las pocas empresas con certificación ambiental en nuestro país, existe un gran número de ellas que, aunque no buscan certificarse, sí utilizan parcialmente las normas y realizan acciones en materia de reciclaje, reutilización, reforestación, conservación de la biodiversidad, etc. Buenas prácticas que no están integradas entre ellas, pero conllevan la mejor de las voluntades por un mejor país.

\section{d. Medición a nivel personal}

La medición de los impactos ambientales a nivel personal va orientada a medir ciertas "huellas" que la persona va dejando conforme a sus hábitos de consumo y su comportamiento dentro de la sociedad. Estos hábitos pueden ser modificados en el tiempo y llegar a ser más responsables.

Cada uno de nosotros debería medir su huella ecológica. Esto es un dato que contempla el consumo de energía, agua, transporte y la generación de residuos con afectación al ambiente. En el caso de la energía, por ejemplo, se llena una encuesta interactiva con datos sobre el número de personas que habitan el hogar, los aparatos electrodomésticos, sistema de iluminación, tipo de cocina y otros, para estimar en kw el empleo de energía en el hogar. La huella personal relativa al agua calcula el porcentaje de consumo de agua en el hogar. La relativa al transporte, brinda unas cifras aproximadas acerca del consumo en transporte y de los kilómetros que se recorren a lo largo de un año. Finalmente, la parte concerniente a los residuos calcula la cantidad de basura generada por persona en el hogar.

Ahora bien, a este nivel lo más importante es que las personas tomen conciencia de sus hábitos de consumo personal dentro y fuera del hogar y de cómo pueden hacer para contribuir con la sostenibilidad ambiental del país. Al 
haber una mayor conciencia, los consumidores son más exigentes con las empresas en cuanto al producto, envase y a la información suministrada en las etiquetas. Ello se traduce luego en una tendencia a utilizar materias primas, procesos de producción y tecnologías más amigables. Aunque todo ello represente un incremento a los precios normales, los consumidores comprometidos con su bienestar y el de la sociedad aceptan de buena gana un incremento moderado. De ahí nace el concepto del marketing verde.

\section{Las nuevas áreas temáticas en la currícula actual}

\section{a. El marketing verde}

El tema del marketing verde puede ser abordado en las carreras de Comercialización, Mercadeo y Licenciatura en Administración de Empresas.

Siendo un concepto relativamente nuevo, el marketing verde permite analizar las estrategias de posicionamiento de productos y servicios en una sociedad cada vez más involucrada con la solución a las problemáticas ambientales concentradas en la biodiversidad, el agua, la energía y los residuos.

Más en detalle las temáticas de segundo orden. Biodiversidad: cuido de especies de flora y fauna, así como ecosistemas amenazados. Agua: contaminación, consumo indiscriminado y falta de saneamiento. Energía: fuentes renovables, el cambio climático, efecto invernadero, energías provenientes de combustibles fósiles, etc. Residuos: Desperdicios, reciclaje, reutilización, contaminación de fuentes hídricas y saturación de rellenos sanitarios.
Consumidores y empresas amenazan continuamente, en diferente forma, el equilibrio de estos ecosistemas mencionados, mediante sus hábitos de consumo y procesos de transformacion. Es vital pensar en que se requiere garantizar la sostenibilidad futura a las nuevas generaciones con una calidad de vida aceptable. Los recursos en la naturaleza son finitos, por lo que se deberán utilizar de manera responsable. No queda otra.

Aunque racionalmente todos estemos de acuerdo con buscar la sostenibilidad ambiental, el reto de implementar las estrategias no es nada fácil. Las empresas deberán trabajar en inducir, con la publicidad y promoción, nuevas pautas de consumo y estilos de vida sostenibles, y reconocer, a partir de la investigación de mercados, el conocimiento de los segmentos de consumidores que están dispuestos a pagar algo más por productos y servicios verdes.

Hay, como siempre, algunas acciones que quedan sólo en apariencias y no en verdaderas estrategias de negocio. Algunas empresas, mal autodenominadas "verdes", se proclaman atributos que no tienen y también hay empresas cuya contribución al medio ambiente (campañas de limpieza, reciclaje interno, etc.) no tienen congruencia con el resto de su quehacer en la práctica.

\section{b. La producción más limpia}

El tema de la producción más limpia puede ser abordado en las carreras de Ingeniería Industrial y Licenciatura en Administración de Empresas.

La producción más limpia, conocida también como PML, es un modelo de producción que busca promover la toma de conciencia, la 
modificación de las actitudes hacia los problemas ambientales mediante la evaluación continua y sistemática de los impactos y opciones tecnológicas para mitigarlos.

Entre las técnicas utilizadas en la producción más limpia se encuentran estos: mejoras en el proceso, establecimiento de buenas prácticas de manufactura (BPM), implementación de enfoques diversos sobre mantenimiento de equipos, reutilización y reciclaje, cambios en la materia prima y diseño de productos, estudios de impacto ambiental en plantas de producción y cambios en la tecnología por otra más eficiente.

Dada la tendencia mundial por los temas ambientales, la aplicación de la PML puede contribuir a las empresas a mejorar su posición competitiva, a reestructurar sus procesos productivos y responder adecuadamente a las normas y certificaciones ambientales requeridas comercialmente en otros mercados.

\section{c. Las fuentes de energía renovable}

Este tema puede ser abordado en las carreras de Ingeniería Eléctrica, Mecánica, Civil, Industrial y en carreras relacionadas con la gestión de recursos como Economía y Licenciatura en Administración de Empresas.

Las fuentes de energía renovables son aquellas que, tras ser utilizadas, se pueden regenerar de manera natural o artificial. Entre ellas tenemos la energía mareomotriz (mareas), hidráulica (embalses y presas), eólica (viento), solar (sol) y biomasa (vegetación) y la obtenida mediante el aprovechamiento del calor del interior de la Tierra (geotérmica).
El estudio de estas fuentes requiere más que una mirada superficial. Son realmente temas complejos que pueden discutirse desde una perspectiva técnica-científica en muchas horasclase estudiando procesos, equipos, cálculos, importancia económica, etc.

\section{d. La logística inversa}

En cuanto al último tema sugerido, este puede ser abordado ampliamente en todas las Ingenierías (Civil, Mecánica, Industrial, Electrónica, etc.), en las carreras de Comercialización y Mercadeo, así como en la Licenciatura en Administración de Empresas. Tiene un amplio rango de acción y se adecúa a muchas de ellas.

La logística inversa (reverse logistics) es un proceso que incluye el movimiento y administración de los productos y recursos desde el cliente hasta el punto final de destrucción del producto. (Ver Figura n. ${ }^{\circ}$ ).

La logística inversa incluye el procesamiento físico de los productos devueltos, que ocurre con frecuencia en un centro de distribución y bodegas, la gestión de las devoluciones pendientes de recibir, la inspección del contenido recibido, la determinación del destino final de los productos y la colocación de los bienes de vuelta al inventario para su reempaque y su correspondiente envío a otras localidades relacionadas o no al negocio, como es el caso de los basureros. Tiene que ver también con la administración de obsoletos, productos fuera de uso, desechos de producción y embalajes, y la gestión de mercados o productos secundarios como lo es venta de productos usados (coches, ropa, equipos, etc.) reacondicionados. 

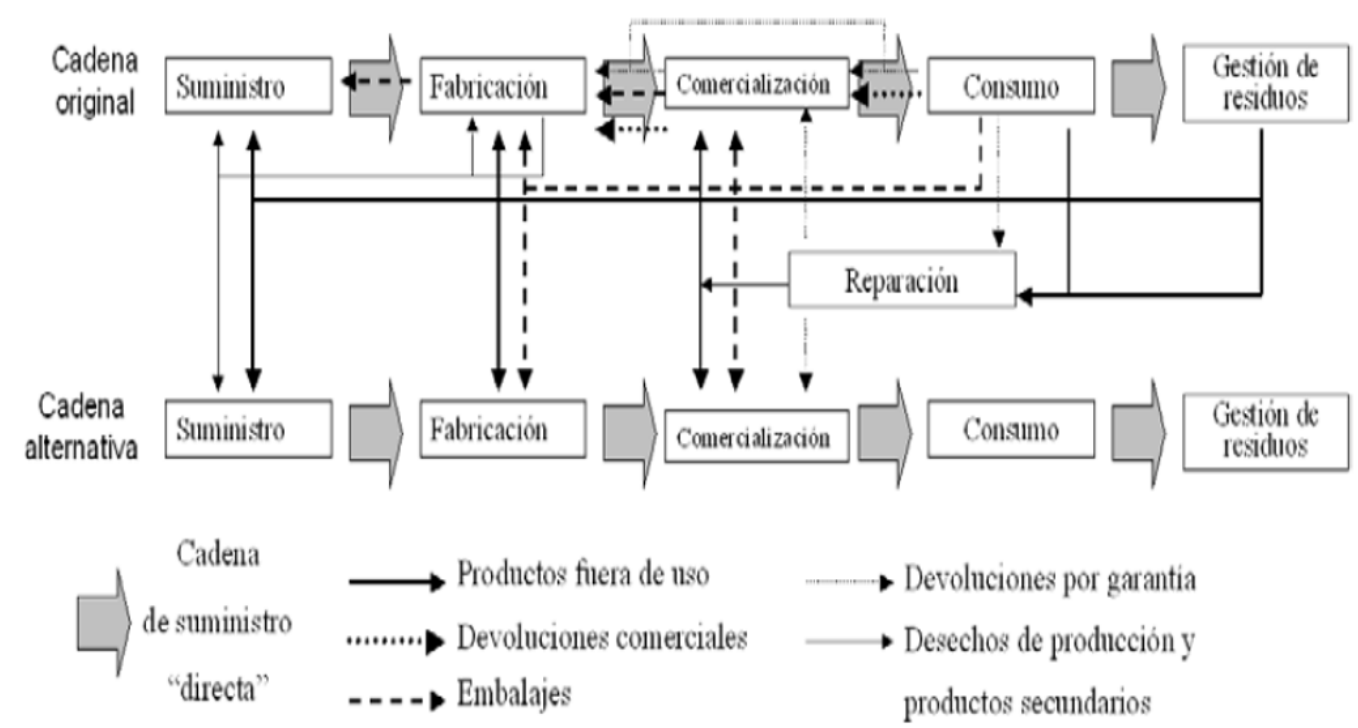

Figura n. ${ }^{3}$. Gestión de la logística inversa y ambiental. Fuente: Luis Mora y María Martín

Dicho de otra forma, el propósito de la logística inversa es la de capturar valor en su disposición final. Involucra el proceso de enviar productos nuevos o usados río arriba (back up stream) para su reparación, reutilización, remanufactura, reventa, reciclado y scrap o, si no es possible extraer valor, entonces pensar en su disposicion final, de acuerdo a la normativa ambiental. Para ejemplificar este fenómeno, se utiliza la pirámide de valor, que indica las opciones habituales para rescatar valor del producto. Se obtiene un máximo valor cuando utilizamos mercados secundarios y el menor valor se obtiene cuando hay que incinerar el producto o enviarlo a rellenos sanitarios. (Ver Figura n. ${ }^{\circ}$ 4).

Tradicionalmente, las empresas no perciben el valor agregado de los flujos inversos (desde el cliente a la empresa por medio de devoluciones y garantías). En algunos casos se les considera "un mal necesario".
Las cadenas de suministro globales representan retos y oportunidades para el flujo inverso de los productos, principalmente en la coordinación de transportes y en el cumplimiento de normativas ambientales y de protección al consumidor.

En la medida en que el sentido de las acciones mostradas en la Figura n. 5 bajan de nivel, el valor que puede extraerse es cada vez menor hasta llegar a la incineración o disposición final en el relleno sanitario.

\section{Conclusiones y recomendaciones}

1. ${ }^{\mathrm{a}}$ El Salvador necesita de una difusión coherente o unificada del análisis técnico de la problemática ambiental. Dada la diversidad de enfoques, las universidades podrían conjuntamente con algún organismo internacional-como la Red Telescopicrear un Observatorio Académico Ambiental 


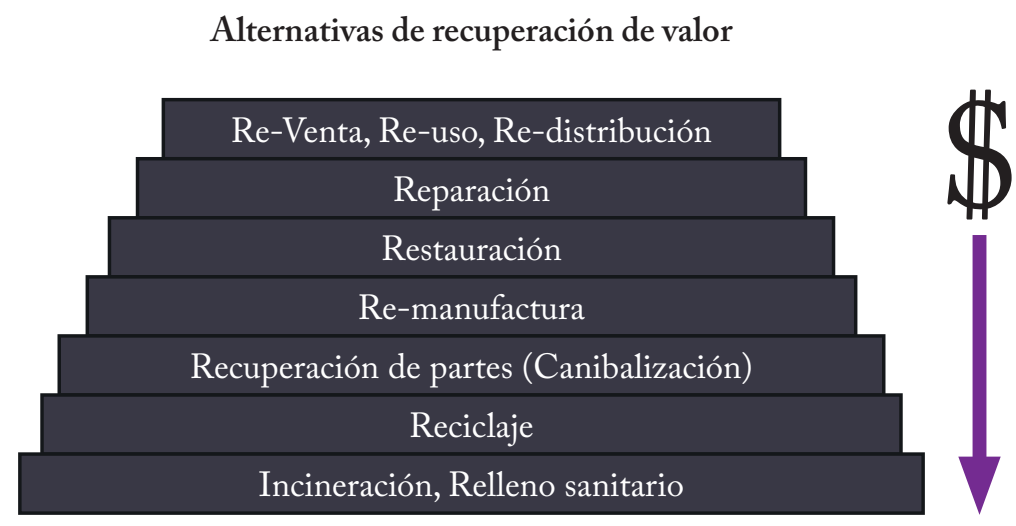

Figura n. ${ }^{\circ}$ 5. Pirámide de valor.

para compartir experiencias, definir contenidos homogéneos a impartir en las universidades y mejorar la calidad docente sobre el tema.

2. ${ }^{\text {a }}$ Dicho Observatorio también tendría facultades para realizar y fomentar investigaciones y consultorías orientadas a mitigar riesgos ambientales en empresas y localidades del país. Ya otros países han avanzado en ello y es preciso realizar esfuerzos coordinados.

3. ${ }^{\text {a }} \mathrm{Y}$ dado que el tema es importante para la sostenibilidad del país y las generaciones futuras, los temas deben abordarse en las carreras de pregrado principalmente con énfasis en el estudio de los temas de marketing verde, producción más limpia, energías renovables y logística inversa. Todo ello permitirá la formación de profesionales más conscientes e integrales. De no hacerse, y de continuar mirando con indiferencia estos temas, no solo se pondría en peligro el bienestar de todos por igual, sino que también se corre el riesgo de contribuir con el aislamiento comercial de nuestro país ante las megatendencias ecológicas que se viven en todo el mundo.

\section{Bibliografía}

Comisión Económica para América Latina y el Caribe (CEPAL). (2016). Bases de datos y publicaciones estadísticas. Recuperado de: http://estadisticas.cepal.org/cepalstat/WEB_ CEPALSTAT/Portada.asp

Fundación Vida Sostenible. (2016). Calculadora personal para buella ecológica. Recuperado de: http://www.vidasostenible.org/

Guía Huella de Carbono http://www.magrama.gob.es/es/cambioclimatico/temas/mitigacion-politicas-y-medidas/ guia_huella_carbono_v2_tcm7-379901.pdf

Huella ecológica Euskadi. (2016). Recuperado de: http://www.tuhuellaecologica.org/

Ihobe. (2016). El sistema de gestión de la mejora ambiental que ahorra dinero a la empresa. Recuperado de: http://www.ihobe.net/Paginas/ Ficha.aspx?IdMenu=bef3bb6c-135a-41fd-bef6129ffeb6881e\&Idioma=es-ES 
Martín García, M. y Mora García, L. (2013). Gestión de la logistica inversa y ambiental: retos $y$ oportunidades en las organizaciones modernas. Bogotá: ECOE Ediciones.

Ministerio de Educación de El Salvador. (2016). Programas educativos. Recuperado de: http:// www.mined.gob.sv/index.php/programaseducativos/educacion-superior

Ministerio de Medio Ambiente y Recursos Naturales (MARN) El Salvador. (2016). Sistema de Evaluación Ambiental. Recuperado de: http:// www.marn.gob.sv/sistema-de-evaluacionambiental-3

Observatorio de Logística y Sustentabilidad ITBA http://itba.edu.ar/es/noticias/elitba-presenta-su-primer-observatorio-de$\log \% \mathrm{C} 3 \% \mathrm{AD}$ stica-y-sustentabilidad
Reverse Logistic Association. Tendencias en logistica inversa. Recuperado de: http://www. reverselogisticstrends.com/

Sistema Nacional de Información Ambiental de México. (2016). Estadísticas ambientales. Recuperado de: http://www.semarnat.gob.mx/ temas/estadisticas-ambientales/snia

Sistema Nacional de Información Ambiental de Perú. (2016). Indicadores ambientales. Recuperado de: http://sinia.minam.gob.pe/estadisticas/ indicadores-ambientales

Universidad de Yale. (2016). Enviroment Performance Index EPI. Recuperado de: http://epi.yale.edu 